\title{
Semi-Commutative Differential Operators Associated with the Dirac Opetator and Darboux Transformation
}

\author{
Masatomo Matsushima, Mayumi Ohmiya* \\ Doshisha University, Kyoto, Japan \\ Email: ${ }^{*}$ momiya@mail.doshisha.ac.jp
}

Received November 20, 2012; revised December 21, 2012; accepted December 29, 2012

\begin{abstract}
In the present paper, the semi-commutative differential oparators associated with the 1-dimensional Dirac operator are constructed. Using this results, the hierarchy of the $\mathrm{mKdV}(-)$ polynomials are expressed in terms of the KdV polynomials. These formulas give a new interpretation of the classical Darboux transformation and the Miura transformation. Moreover, the recursion operator associated with the hierarchy of the $\mathrm{mKdV}(-)$ polynomials is constructed by the algebraic method.
\end{abstract}

Keywords: KdV Polynomials; mKdV (-) Polynomials; Schrödinger Operator; Dirac Operator

\section{Introduction}

The main purpose of the present paper ${ }^{1}$ is to construct the semi-commutative differential operators associated with the 1-dimensional Dirac operator

$$
P(v)=\left(\begin{array}{cc}
0 & 1 \\
-1 & 0
\end{array}\right) D+\left(\begin{array}{cc}
0 & v(x) \\
v(x) & 0
\end{array}\right), D=\frac{\mathrm{d}}{\mathrm{d} x},
$$

where the potential $v=v(x)$ is the infinitely differentiable function. Define the 1st order ordinary differential operartors by

$$
L^{ \pm}(v)= \pm D+v(x),
$$

then, the oprtator $P(v)$ is expressed as

$$
P(v)=\left(\begin{array}{cc}
0 & L^{+}(v) \\
L^{-}(v) & 0
\end{array}\right) .
$$

Note that the variable $x$ can be regarded as both real or complex throughout the paper.

The differential operators $A, B$ are said to be semicommutative, if the commutator $[A, B]=A B-B A$ is the multiplicative operator. As for the 1-dimensional Schrödinger operator

$$
H(u)=-D^{2}+u(x),
$$

the identities

$$
\left[A_{n}(u), H(u)\right]=\frac{\mathrm{d}}{\mathrm{d} x} Z_{n+1}(u), n \in N
$$

\footnotetext{
"Corresponding author.

${ }^{1}$ This work was supported by JSPS KAKENHI Grant Number 23540255 .
}

are well known for the differential operator $A_{n}(u)$ of the order $2 n+1$ difined by

$$
A_{n}(u)=\frac{1}{2} \sum_{j=0}^{n}\left(Z_{j}(u) D-\frac{1}{2} \frac{\mathrm{d}}{\mathrm{d} x} Z_{j}(u)\right) H(u)^{n-j},
$$

where $Z_{j}(u)$ are the $\mathrm{KdV}$ polynomials which will be explained precisely in Section 2.

On the other hand, in [1], R. M. Miura discovered the following interesting fact; if $v=v(x, t)$ solves the $\mathrm{mKdV}(-)$ equation

$$
v_{t}-6 v^{2} v_{x}+v_{x x x}=0,
$$

then both functions $u^{ \pm}=u^{ \pm}(x, t)$ defined by

$$
u^{ \pm}(x, t)= \pm v_{x}(x, t)+v(x, t)^{2}
$$

solve the $\mathrm{KdV}$ equation

$$
u_{t}-6 u u_{x}+u_{x x x}=0,
$$

where the subscript denotes the partial differentiation. The transformation defined by (7) is the Miura transformation which plays the crucial role in the soliton theory. By (7), we have immediately the relation

$$
u^{+}=u^{-}-2 v_{x} .
$$

The transformation $u^{+} \mapsto u^{-}$defined by (9) is nothing but the Darboux transformation. Using the $\mathrm{KdV}$ polynomial, the relation (9) can be expressed as

$$
Z_{1}\left(u^{-}\right)-Z_{1}\left(u^{+}\right)=v_{x} .
$$

Considering the above facts, we investigate the problem to express the differential polynomials 
$Z_{j}\left(u^{-}\right)-Z_{j}\left(u^{+}\right)$in terms of the function $v=v(x)$ for the stationary case, i.e., when the function $v$ is independent of the time variable $t$, i.e., in what follows $u^{ \pm}(x)$ are defined by

$$
u^{ \pm}(x)= \pm v^{\prime}(x)+v(x)^{2} .
$$

As a result, we obtain the new formulation of the stationary $\mathrm{mKdV}(-)$ hierarchy.

In our previous works [2,3], it is clarified that the semi-commutative operators and Darboux transformation are deeply related to the spectral theory of the differential operator $H(u)$ when the potential $u=u(x)$ is algebrogeometric. The aim of our work is to extend these results concerned with the 1-dimensional Schrödinger operator $H(u)$ to the 1-dimensional Dirac operator $P(v)$. The present work can be regarded as the first step of it. By applying the results of the present paper, we can obtain the various transformation formulas concerned with the algebro-geometric elliptic potential. These results will be reported in the forthcoming paper.

The contents of the present paper are as follows. In Section 2, we explain the fundamental materials which are necessary for the present work. In Section 3, calculation of the commutator of the Dirac operator and the differential operator constructed from the semi-commutative operator of the KdV hierarchy is carried out, and the main theorem of the present paper is stated. Section 4 is devoted to the proof of the main theorem. In Section 5, we construct the recursion operator associated with the $\mathrm{mKdV}$ hierarchy.

\section{Preliminaries}

The KdV polynomials $Z_{n}(u)$ are differential polynomials defined by the recurrence relation

$$
Z_{n}(u)=\Lambda(u) Z_{n-1}(u), n=1,2, \cdots,
$$

with the condition $Z_{0}(u)=1$, where $\Lambda(u)$ is the formal pseudo-differential operator defined by

$$
\Lambda(u)=D^{-1}\left(\frac{1}{2} u^{\prime}(x)+u(x) D-\frac{1}{4} D^{3}\right) .
$$

Then $Z_{n}(u)$ turn out to be the differential polynomials in $u(x)$. For examples, we have

$$
Z_{1}(u)=\frac{1}{2} u, Z_{2}(u)=\frac{3}{8} u^{2}-\frac{1}{8} u^{\prime \prime} .
$$

If $u=u(x, t)$ depends also on the time variable $t$, then the evolution equation

$$
\frac{\partial u}{\partial t}=\frac{\partial}{\partial x} Z_{2}(u)=\frac{3}{4} u \frac{\partial u}{\partial x}-\frac{1}{8} \frac{\partial^{3} u}{\partial x^{3}}
$$

is nothing but the $\mathrm{KdV}$ equation. Hence, we call them the $K d V$ polynomials. See [3] for more details of the $\mathrm{KdV}$ polynomials.

In what follows, we will often use the higher order derivatives of the differential polynomials $Z_{j}(u)$. So, for the brevity, we will use the following notations of derivatives of the $\mathrm{KdV}$ polynomials defined by

$$
X_{j}^{(k)}(u)=\frac{\mathrm{d}^{k}}{\mathrm{~d} x^{k}} Z_{j}(u), k=0,1,2, \cdots,
$$

where $X_{j}^{(0)}(u)=Z_{j}(u)$. Thus we have

$$
\left[A_{n}(u), H(u)\right]=X_{n+1}^{(1)}(u)
$$

From now on, we restrict ourselves to the stationary problem, i.e., the function $v=v(x)$ under consideration depends only on the space variable $x$.

One immediately verifies the identities

$$
H\left(u^{ \pm}\right)=L^{ \pm}(v) L^{\mp}(v) .
$$

Define the multi-component operator $S(v)$ by

$$
\begin{aligned}
S(v) & =\left(\begin{array}{cc}
H\left(u^{+}\right) & 0 \\
0 & H\left(u^{-}\right)
\end{array}\right) \\
& =-\left(\begin{array}{ll}
1 & 0 \\
0 & 1
\end{array}\right) D^{2}+\left(\begin{array}{cc}
u^{+}(x) & 0 \\
0 & u^{-}(x)
\end{array}\right) .
\end{aligned}
$$
tity

By (13), one can show immediately the operator iden-

$$
P(v)^{2}=\left(\begin{array}{cc}
L^{+}(v) L^{-}(v) & 0 \\
0 & L^{-}(v) L^{+}(v)
\end{array}\right)=S(v) .
$$

On the other hand, define the multi-component differential operator $\tilde{A}_{n}(v)$ by

$$
\tilde{A}_{n}(v)=\left(\begin{array}{cc}
A_{n}\left(u^{+}\right) & 0 \\
0 & A_{n}\left(u^{-}\right)
\end{array}\right) .
$$

Then, by (5), (14), and (16), we have immediately

$$
\begin{aligned}
& {\left[\tilde{A}_{n}(v), S(v)\right]} \\
& =\left(\begin{array}{cc}
{\left[A_{n}\left(u^{+}\right), H\left(u^{+}\right)\right]} & 0 \\
0 & {\left[A_{n}\left(u^{-}\right), H\left(u^{-}\right)\right]}
\end{array}\right) \\
& =\left(\begin{array}{cc}
X_{n+1}^{(1)}\left(u^{+}\right) & 0 \\
0 & X_{n+1}^{(1)}\left(u^{-}\right)
\end{array}\right) .
\end{aligned}
$$

Therefore the operator $\tilde{A}_{n}(v)$ are semi-commutative with the operator $S(v)$.

\section{Calculation of $\left[\tilde{A}_{n}(v), P(v)\right]$}

Define the scalar differential operators $K_{n}^{ \pm}(v)$ by

$$
K_{n}^{ \pm}(v)=A_{n}\left(u^{\mp}\right) L^{\mp}(v)-L^{\mp}(v) A_{n}\left(u^{ \pm}\right)
$$

respectively. By direct calculation, we have immediately 


$$
\begin{aligned}
{\left[\tilde{A}_{n}(v), P(v)\right] } & =\tilde{A}_{n}(v) P(v)-P(v) \tilde{A}_{n}(v) \\
& =\left(\begin{array}{cc}
0 & K_{n}^{-}(v) \\
K_{n}^{+}(v) & 0
\end{array}\right) .
\end{aligned}
$$

By the definition (6) of the operator $A_{n}(u)$, we have immediately

$$
\begin{aligned}
K_{n}^{ \pm}(v)= & \frac{1}{2} \sum_{j=0}^{n}\left\{\left(X_{j}^{(0)}\left(u^{\mp}\right) D-\frac{1}{2} X_{j}^{(1)}\left(u^{\mp}\right)\right) L^{\mp}(v)\right. \\
& \left.-L^{\mp}(v)\left(X_{j}^{(0)}\left(u^{ \pm}\right) D-\frac{1}{2} X_{j}^{(1)}\left(u^{ \pm}\right)\right)\right\} H\left(u^{ \pm}\right)^{n-j}
\end{aligned}
$$

By (15), we have

$$
\begin{aligned}
H\left(u^{ \pm}\right)^{n-j} L^{ \pm}(v) & =\left(L^{ \pm}(v) L^{\mp}(v)\right)^{n-j} L^{ \pm}(v) \\
& =L^{ \pm}(v)\left(L^{\mp}(v) L^{ \pm}(v)\right)^{n-j} \\
& =L^{ \pm}(v) H\left(u^{\mp}\right)^{n-j} .
\end{aligned}
$$

Furthermore, by the definition (2) of $L^{ \pm}(v)$, one verifies

$$
\begin{aligned}
K_{n}^{ \pm}(v)= & \frac{1}{2} \sum_{j=0}^{n}\left\{\left(X_{j}^{(0)}\left(u^{+}\right)-X_{j}^{(0)}\left(u^{-}\right)\right) D^{2}\right. \\
& \pm \frac{1}{2} v\left(X_{j}^{(1)}\left(u^{+}\right)-X_{j}^{(1)}\left(u^{-}\right)\right) \\
& \left.+v^{\prime} X_{j}^{(0)}\left(u^{\mp}\right) \mp \frac{1}{2} X_{j}^{(2)}\left(u^{ \pm}\right)\right\} H\left(u^{ \pm}\right)^{n-j},
\end{aligned}
$$

where we used the identity

$$
B^{-} Z_{j}\left(u^{+}\right)=B^{+} Z_{j}\left(u^{-}\right),
$$

where

$$
B^{ \pm}= \pm D+2 v .
$$

The identity (22) is derived in [4], and is called the fundamental identity of the Darboux transformation in it, By the fundamental identity (22), we have immediately

$$
\begin{aligned}
& \left\{\mp v\left(X_{j}^{(0)}\left(u^{+}\right)-X_{j}^{(0)}\left(u^{-}\right)\right)\right. \\
& \left. \pm \frac{1}{2}\left(X_{j}^{(1)}\left(u^{+}\right)+X_{j}^{(1)}\left(u^{-}\right)\right)\right\} D=0 .
\end{aligned}
$$

Put

$$
\begin{aligned}
J_{n}^{ \pm}(v)= & \frac{1}{2}\left\{\left(X_{n}^{(0)}\left(u^{+}\right)-X_{n}^{(0)}\left(u^{-}\right)\right) D^{2}\right. \\
& \pm \frac{1}{2} v\left(X_{n}^{(1)}\left(u^{+}\right)-X_{n}^{(1)}\left(u^{-}\right)\right) \\
& \left.+v^{\prime} X_{n}^{(0)}\left(u^{\mp}\right) \mp \frac{1}{2} X_{n}^{(2)}\left(u^{ \pm}\right)\right\},
\end{aligned}
$$

then we can express the identity (21) in terms of $J_{n}^{ \pm}(v)$ as

$$
K_{n}^{ \pm}(v)=J_{n}^{ \pm}(v)+K_{n-1}^{ \pm}(v) H\left(u^{ \pm}\right), n=0,1,2, \cdots .
$$

For $n=0,1$, one verifies easily

$$
\begin{gathered}
K_{0}^{ \pm}(v)=\frac{1}{2} v^{\prime}, \\
K_{1}^{ \pm}(v)=-\frac{1}{8} v^{\prime \prime \prime}+\frac{3}{4} v^{2} v^{\prime} .
\end{gathered}
$$

Thus, for $n=0,1, K_{n}^{+}(v)=K_{n}^{-}(v)$ hold, and they are not differential operators, but are the multipricative operator. Thus, the operator $\tilde{A}_{n}(u)$ and the Dirac operator $P(v)$ turns out to be semi-commutative for $n=0,1$.

For general $n$, we have the following theorem which is the main result of the present paper.

Theorem 1. The multi-component differential operators $\tilde{A}_{n}(v)$ and $P(v)$ are semi-commutative, i.e., $K_{n}^{ \pm}(v)$ are the multiplicative operators, and they coincide with each other, i.e., the equality

$$
K_{n}^{+}(v)=K_{n}^{-}(v)
$$

hold for all $n$. Moreover, if we denote them as $K_{n}(v)$, then the identities

$$
K_{n}(v)=\frac{1}{2}\left(Z_{n+1}\left(u^{+}\right)-Z_{n+1}\left(u^{-}\right)\right)
$$

hold for all $n \in Z_{+}=N \cup\{0\}$.

If the potential $v$ depends also on the time variable $t$, i.e., $v=v(x, t)$, the evolution equation

$$
\frac{\partial v}{\partial t}=K_{1}(v)=-\frac{1}{8} \frac{\partial^{3} v}{\partial x^{3}}+\frac{3}{4} v^{2} \frac{\partial v}{\partial x}
$$

is nothing but the $\mathrm{mKdV}(-)$ equation. Hence, we call the differential polynomials $K_{n}(v), n \in Z_{+}=N \cup\{0\}$ the $m K d V(-)$ polynomials.

\section{The Proof of Theorem 1}

We prove the theorem by induction. Firstly, for $n=0$, one verifies

$$
K_{0}^{ \pm}(v)=\frac{1}{2} v^{\prime} .
$$

On the other hand, by (10), we have

$$
\begin{aligned}
\frac{1}{2}\left(X_{1}^{(0)}\left(u^{+}\right)-X_{1}^{(0)}\left(u^{-}\right)\right) & =\frac{1}{2}\left(\frac{1}{2} u^{+}-\frac{1}{2} u^{-}\right) \\
& =\frac{1}{2}\left(\frac{1}{2}\left(v^{\prime}+v^{2}\right)-\frac{1}{2}\left(-v^{\prime}+v^{2}\right)\right) \\
& =\frac{1}{2} v^{\prime}=K_{0}^{ \pm}(v) .
\end{aligned}
$$

Secondly, for $n-1$, we assume that 


$$
K_{n-1}^{ \pm}(v)=\frac{1}{2}\left(X_{n}^{(0)}\left(u^{+}\right)-X_{n}^{(0)}\left(u^{-}\right)\right)
$$

holds. Then, for $n$, we have

$$
K_{n}^{ \pm}(v)=J_{n}^{ \pm}(v)+\frac{1}{2}\left(X_{n}^{(0)}\left(u^{+}\right)-X_{n}^{(0)}\left(u^{-}\right)\right) H\left(u^{ \pm}\right) .
$$

From (21) and (23), we have

$$
\begin{aligned}
K_{n}^{ \pm}(v)= & \frac{1}{2}\left( \pm \frac{1}{2} v X_{n}^{(1)}\left(u^{+}\right) \mp \frac{1}{2} v X_{n}^{(1)}\left(u^{-}\right)+v^{2} X_{n}^{(0)}\left(u^{+}\right)\right. \\
& \left.-v^{2} X_{n}^{(0)}\left(u^{-}\right) \mp \frac{1}{2} X_{n}^{(2)}\left(u^{ \pm}\right)+v^{\prime} X_{n}^{(0)}\left(u^{ \pm}\right)\right) .
\end{aligned}
$$

This implies that $K_{n}^{ \pm}(v)$ are the multiplicative operators, i.e., $\tilde{A}_{n}(v)$ and $P(v)$ are semi-commutative.

Similarly, by induction, we can show that $K_{n}^{ \pm}(v)$ coincide with each other.

Next we show the identity (26). By straightforward calculation, one can show

$$
\begin{aligned}
& \frac{\mathrm{d}}{\mathrm{d} x} K_{n}^{ \pm}(v)=\frac{1}{2}\left(\mp \frac{1}{2} X_{n}^{(3)}\left(u^{ \pm}\right) \pm \frac{1}{2} v X_{n}^{(2)}\left(u^{+}\right)\right. \\
& \mp \frac{1}{2} v X_{n}^{(2)}\left(u^{-}\right)-\frac{1}{2} v^{\prime} X_{n}^{(1)}\left(u^{\mp}\right)+\frac{3}{2} v^{\prime} X_{n}^{(1)}\left(u^{ \pm}\right) \\
& +v^{2} X_{n}^{(1)}\left(u^{+}\right)-v^{2} X_{n}^{(1)}\left(u^{-}\right)+2 v v^{\prime} X_{n}^{(0)}\left(u^{+}\right) \\
& \left.-2 v v^{\prime} X_{n}^{(0)}\left(u^{-}\right)+v^{\prime \prime} X_{n}^{(0)}\left(u^{ \pm}\right)\right) .
\end{aligned}
$$

Put

$$
K_{n}(v)=\frac{1}{2}\left(K_{n}^{+}(v)+K_{n}^{-}(v)\right),
$$

then, by (27), we have

$$
\begin{aligned}
& \frac{\mathrm{d}}{\mathrm{d} x} K_{n}(v)=\frac{1}{2}\left(\frac{\mathrm{d}}{\mathrm{d} x} K_{n}^{+}(v)+\frac{\mathrm{d}}{\mathrm{d} x} K_{n}^{-}(v)\right) \\
& =\frac{1}{2}\left(-\frac{1}{4} X_{n}^{(3)}\left(u^{+}\right)+\frac{1}{4} X_{n}^{(3)}\left(u^{-}\right)+\frac{1}{2} v^{\prime} X_{n}^{(1)}\left(u^{+}\right)\right. \\
& \quad+\frac{1}{2} v^{\prime} X_{n}^{(1)}\left(u^{-}\right)+v^{2} X_{n}^{(1)}\left(u^{+}\right)-v^{2} X_{n}^{(1)}\left(u^{-}\right) \\
& \quad+\frac{1}{2} v^{\prime \prime} X_{n}^{(0)}\left(u^{+}\right)+\frac{1}{2} v^{\prime \prime} X_{n}^{(0)}\left(u^{-}\right) \\
& \left.\quad+2 v v^{\prime} X_{n}^{(0)}\left(u^{+}\right)-2 v v^{\prime} X_{n}^{(0)}\left(u^{-}\right)\right),
\end{aligned}
$$

where we used the relation

$$
\begin{aligned}
& \frac{1}{2} v^{\prime} X_{n}^{(1)}\left(u^{+}\right)+\frac{1}{2} v^{\prime} X_{n}^{(1)}\left(u^{-}\right) \\
& =v v^{\prime} X_{n}^{(0)}\left(u^{+}\right)-v v^{\prime} X_{n}^{(0)}\left(u^{-}\right)
\end{aligned}
$$

which are derived immediately from (22).

Thus, we have

$$
\begin{aligned}
\frac{\mathrm{d}}{\mathrm{d} x} K_{n}(v)= & \frac{1}{2}\left\{\frac{1}{2}\left(v^{\prime \prime}+2 v v^{\prime}\right) X_{n}^{(0)}\left(u^{+}\right)\right. \\
& +\left(v^{\prime}+v^{2}\right) X_{n}^{(1)}\left(u^{+}\right)-\frac{1}{4} X_{n}^{(3)}\left(u^{+}\right) \\
& -\frac{1}{2}\left(-v^{\prime \prime}+2 v v^{\prime}\right) X_{n}^{(0)}\left(u^{-}\right) \\
& \left.-\left(-v^{\prime}+v^{2}\right) X_{n}^{(1)}\left(u^{-}\right)+\frac{1}{4} X_{n}^{(3)}\left(u^{-}\right)\right\} .
\end{aligned}
$$

By (7), we have

$$
\begin{aligned}
\frac{\mathrm{d}}{\mathrm{d} x} K_{n}(v)= & \frac{1}{2}\left\{\left(\frac{1}{2} u_{x}^{+}(x)+u^{+}(x) D-\frac{1}{4} D^{3}\right) X_{n}^{(0)}\left(u^{+}\right)\right. \\
& \left.-\left(\frac{1}{2} u_{x}^{-}(x)+u^{-}(x) D-\frac{1}{4} D^{3}\right) X_{n}^{(0)}\left(u^{-}\right)\right\}
\end{aligned}
$$

By (12) which is the definition of $\Lambda(u)$, we have

$$
\begin{aligned}
K_{n}(v) & =\frac{1}{2}\left(\Lambda\left(u^{+}\right) X_{n}^{(0)}\left(u^{+}\right)-\Lambda\left(u^{-}\right) X_{n}^{(0)}\left(u^{-}\right)\right) \\
& =\frac{1}{2}\left(Z_{n+1}\left(u^{+}\right)-Z_{n+1}\left(u^{-}\right)\right) .
\end{aligned}
$$

This completes the proof of Theorem 1.

\section{The Recursion Operator}

In the preceding section, we have shown the relation

$$
K_{n}(v)=\frac{1}{2}\left(Z_{n+1}\left(u^{+}\right)-Z_{n+1}\left(u^{-}\right)\right)
$$

hold for all $n$. Therefore, by (28), we have

$$
\begin{aligned}
K_{n}(v)=\frac{1}{2}\left\{2 v v^{\prime} K_{n-1}+2 v^{2} K_{n-1}^{\prime}-\frac{1}{2} K_{n-1}^{\prime \prime \prime}\right. \\
+\frac{1}{2} v^{\prime \prime}\left(X_{n}^{(0)}\left(u^{+}\right)+X_{n}^{(0)}\left(u^{-}\right)\right) \\
\left.+v^{\prime}\left(X_{n}^{(1)}\left(u^{+}\right)+X_{n}^{(1)}\left(u^{-}\right)\right)\right\} .
\end{aligned}
$$

Then, by (22), we have

$$
\begin{aligned}
K_{n}= & D^{-1}\left(-\frac{1}{4} K_{n-1}^{\prime \prime \prime}+v^{2} K_{n-1}^{\prime}+2 v v^{\prime} K_{n-1}\right) \\
& +v^{\prime} D^{-1}\left(v K_{n-1}\right)
\end{aligned}
$$

The relation (29) defines the recursion operators of $K_{n}(v)$. Therefore, we have the following theorem.

Theorem 2. The formal pseudo-differential operator $\Gamma(v)$ defined by

$$
\Gamma(v) f=D^{-1}\left(-\frac{1}{4} D^{3} f+v^{2} D f+2 v v^{\prime} f\right)+v^{\prime} D^{-1}(v f)
$$

is the recursion operator associated with the $\mathrm{mKdV}(-)$ polynomials $K_{n}(v), n \in \mathbf{Z}_{+}=N \bigcup\{0\}$, i.e., 


$$
\Gamma(v) K_{n-1}(v)=K_{n}(v), n \in N
$$

hold.

Using this recursion operator $\Gamma(v)$, one can calculate easily the hierarchy of the $\mathrm{mKdV}(-)$ polynomials.

\section{REFERENCES}

[1] R. M. Miura, "Korteweg-De Vries Equation and Generalizations. I. A Remarkable Explicit Nonlinear Transformation," Journal of Mathematical Physics, Vol. 9, No. 8, 1968, pp. 1202-1204. doi:10.1063/1.1664700

[2] M. Ohmiya, "Spectrum of Darboux Transformation of
Differential Operator," Osaka Journal of Mathematics, Vol. 36, No. 4, 1999, pp. 949-980.

[3] M. Ohmiya, "KdV Polynomials and $\Lambda$-Operator," Osaka Journal of Mathematics, Vol. 32, No. 2, 1995, pp. 409430.

[4] M. Ohmiya and Y. P. Mishev, "Darboux Transformation and $\Lambda$-Operator," Journal of Mathematics/Tokushima University, Vol. 27, 1993, pp. 1-15.

[5] M. Matsushima and M. Ohmiya, "An Algebraic Construction of the First Integrals of the Stationary KdV Hierarchy," Proceeding of ICNAAM: Numerical Analysis and Applied Mathematics, Vol. 1, 2009, pp. 168-172. 